Elect. Comm. in Probab. 5 (2000) 223-234

\title{
ALMOST SURE STABILITY OF LINEAR ITÔ-VOLTERRA EQUA- TIONS WITH DAMPED STOCHASTIC PERTURBATIONS
}

\author{
JOHN A. D. APPLEBY \\ School of Mathematical Sciences, \\ Dublin City University, Dublin 9, Ireland \\ email: john.appleby@dcu.ie
}

submitted May 23, 2002 Final version accepted 21 August 2002

AMS 2000 Subject classification: Primary: 60H10, 60H20, 34K20. Secondary: 45D05.

stochastic functional-differential equations, Itô-Volterra equations, uniform asymptotic stability, almost sure stability, pathwise stability, simulated annealing

Abstract

In this paper we study the a.s. convergence of all solutions of the Itô-Volterra equation

$$
d X(t)=\left(A X(t)+\int_{0}^{t} K(t-s) X(s) d s\right) d t+\Sigma(t) d W(t)
$$

to zero. $A$ is a constant $d \times d$ matrix, $K$ is a $d \times d$ continuous and integrable matrix function, $\Sigma$ is a continuous $d \times r$ matrix function, and $W$ is an $r$-dimensional Brownian motion. We show that when

$$
x^{\prime}(t)=A x(t)+\int_{0}^{t} K(t-s) x(s) d s
$$

has a uniformly asymptotically stable zero solution, and the resolvent has a polynomial upper bound, then $X$ converges to 0 with probability 1 , provided

$$
\lim _{t \rightarrow \infty}\|\Sigma(t)\|^{2} \log t=0
$$

A converse result under a monotonicity restriction on $\|\Sigma\|$ establishes that the rate of decay for $\|\Sigma\|$ above is necessary. Equations with bounded delay and neutral equations are also considered.

\section{INTRODUCTION}

A literature has appeared over the last fifteen years on the properties of linear stochastic functional equations with additive noise (often supplied by Brownian motion). See for instance, Küchler and Mensch [7], Mohammed and Scheutzow [12], and work in Mohammed [11]. Such evolutions have Gaussian solutions which can be represented in a variation of parameters form. The similarity of this representation to that of the solutions of linear stochastic differential equations with deterministic volatility indicates that a steady state will be reached by the solutions of such equations under similar conditions that bring about the a.s. stability of the solution of the SDE. However, the economical proof which suffices in the SDE case is impossible 
to imitate owing to the impossibility of writing the random contribution as a product of a deterministic (matrix) function and a martingale.

A strand of recent research in the stability of stochastic equations with delay has focussed on the speed at which convergence to an equilibrium may take place in the presence of such damped external perturbations. We highlight here the contribution of Mao [8], and Mao and Liao [9]. In these papers, the exponential stability of solutions is considered. In this paper, we ask how large damped stochastic perturbations can be, while still allowing convergence at any speed to the equilibrium. The motivation in these papers, as here, is to ask whether an asymptotically stable deterministic system will remain (almost surely) asymptotically stable when it is subjected to an external random perturbation whose intensity decays over time. In later work, we return to consider necessary and sufficient conditions under which linear and nonlinear Itô-Volterra equations with damped stochastic perturbations exhibit a.s. exponential stability.

We study the simpler linear problem, exploiting the Gaussianity of the solution, in an attempt to obtain necessary and sufficient conditions on the rate of decay of the noise intensity under which the solutions converge a.s. to equilibrium. We are motivated to determine sharp conditions on the rate of decay of the noise perturbation by results of Chan and Williams in [4] for stochastic differential equations in the study of simulated annealing.

To obtain sufficient conditions for convergence, we suppose that the underlying deterministic system has a uniformly asymptotically stable equilibrium, and a polynomially bounded resolvent. It transpires that if the coefficient of the volatility matrix decay to 0 faster than $1 / \sqrt{\log t}$ as $t \rightarrow \infty$, then the solution of the Itô-Volterra equation converges to 0 a.s.

The result of the theorem is also shown to be quite sharp. Under a monotonicity constraint on the norm of the matrix $\Sigma$, one can show that the a.s. asymptotic stability of solutions of the linear Itô-Volterra equation implies that the norm of the volatility matrix decays faster than $1 / \sqrt{\log t}$, as $t \rightarrow \infty$, which was earlier seen to be sufficient for almost sure asymptotic stability. In a final section, we mention that the results may be easily extended to linear equations with bounded delay, and also to neutral equations.

\section{Supporting Results}

We first fix some standard notation. As usual, let $x \vee y$ denote the maximum of $x, y \in \mathbb{R}$, and $x \wedge y$ their minimum.

Denote by $C(I ; J)$ the space of continuous functions taking the finite dimensional Banach space $I$ onto the finite dimensional Banach space $J$. Let $d$ be a positive integer. Let $\left(\mathbb{R}^{d}\right)^{n}$ be the $n$-fold Cartesian product of $\mathbb{R}^{d}$ with itself. Let $M_{d, d}(\mathbb{R})$ denote the space of all $d \times d$ matrices with real entries, and $C\left(\mathbb{R}^{+} ; M_{d, d}(\mathbb{R})\right)$ stand for all continuous $d \times d$ matrix-valued functions with domain $\mathbb{R}^{+}$. The corresponding $d \times r$ matrices and matrix functions lie in the spaces $M_{d, r}(\mathbb{R})$ and $C\left(\mathbb{R}^{+} ; M_{d, r}(\mathbb{R})\right)$, respectively. We say that the function $f: \mathbb{R}^{+} \rightarrow M_{n, m}(\mathbb{R})$ is in $L^{1}\left(\mathbb{R}^{+}\right)\left(\right.$resp. $L^{2}\left(\mathbb{R}^{+}\right)$) if each of its entries is a scalar Lebesgue integrable function (resp. a scalar square integrable Lebesgue function). The transpose of any matrix $A$ is denoted $A^{T}$; the trace of a square matrix is denoted by $\operatorname{tr}(A)$. Further denote by $I_{d}$ the identity matrix in $M_{d, d}(\mathbb{R})$. Let $\|x\|$ stand for the Euclidean norm of $x \in \mathbb{R}^{d}$, and $\|x\|_{1}$ be the sum of the absolute values of the components of $x$. If $A=\left(A_{i j}\right) \in M_{d, r}(\mathbb{R}), A$ has operator norm denoted by $\|A\|$, and given by

$$
\|A\|=\sup \left\{\|A x\|: x \in \mathbb{R}^{r},\|x\|=1\right\} .
$$


It further has Frobenius norm, denoted by $\|A\|_{F}$, and defined as follows: if $A=\left(a_{i, j}\right)$, is an $d \times r$ matrix, then

$$
\|A\|_{F}=\left(\sum_{i=1}^{d} \sum_{j=1}^{r}\left|a_{i, j}\right|^{2}\right)^{1 / 2}=\operatorname{tr}\left(A A^{T}\right)^{\frac{1}{2}} .
$$

Since $M_{d, r}(\mathbb{R})$ is a finite dimensional Banach space, $\|\cdot\|,\|\cdot\|_{F}$ are equivalent, so there exist positive universal constants $c_{1}(d, r) \leq c_{2}(d, r)$ such that

$$
c_{1}(d, r)\|A\| \leq\|A\|_{F} \leq c_{2}(d, r)\|A\|, \quad A \in M_{d, r}(\mathbb{R}) .
$$

For $\phi \in C\left(\left[0, t_{0}\right] ; \mathbb{R}^{d}\right)$, define $|\phi|_{t_{0}}=\sup \left\{\|\phi(t)\|_{1}: 0 \leq t \leq t_{0}\right\}$.

We first turn our attention to the deterministic Volterra equation

$$
x^{\prime}(t)=A x(t)+\int_{0}^{t} K(t-s) x(s) d s, \quad t \geq 0
$$

where $A \in M_{d, d}(\mathbb{R}), K(t) \in C\left(\mathbb{R}^{+} ; M_{d, d}(\mathbb{R})\right)$ and $K \in L^{1}\left(\mathbb{R}^{+}\right)$. For any $t_{0} \geq 0$ and $\phi \in$ $C\left(\left[0, t_{0}\right] ; \mathbb{R}^{d}\right)$, there is a unique $\mathbb{R}^{d}$-valued function $x(t)$, which satisfies $(2.1)$ on $\left[t_{0}, \infty\right)$ and for which $x(t)=\phi(t)$ for $t \in\left[0, t_{0}\right]$. We denote such a solution by $x\left(t ; t_{0}, \phi\right)$. The function $x(t) \equiv 0$ is a solution of (2.1) and is called the zero solution of (2.1).

Consider now the matrical equation

$$
Z^{\prime}(t)=A Z(t)+\int_{0}^{t} K(t-s) Z(s) d s, \quad t \geq 0
$$

with $Z(0)=I_{d}$. The unique $Z \in C\left(\mathbb{R}^{+} ; M_{d, d}(\mathbb{R})\right)$ which satisfies $(2.2)$ is called the resolvent, or principal matrix solution for $(2.1)$.

Existence and uniqueness results for deterministic Volterra equations are covered in Driver [5]. In this paper, we will consider $d$-dimensional linear stochastic integro-differential equations with stochastic perturbations of the form

$$
d X(t)=\left(A X(t)+\int_{0}^{t} K(t-s) X(s) d s\right) d t+\Sigma(t) d W(t)
$$

on $t \geq 0$ where $(W(t))_{t \geq 0}$ is an $r$-dimensional Brownian motion on a complete filtered probability space $\left(\Omega, \mathcal{F},\left(\mathcal{F}_{t}\right)_{t \geq 0}, \mathbb{P}\right)$, where the filtration is the natural one $\mathcal{F}_{t}=\sigma\{W(s): 0 \leq s \leq t\}$. $X$ has deterministic initial condition $X_{0}=x, A \in M_{d, d}(\mathbb{R}), \Sigma \in C\left(\mathbb{R}^{+}, M_{d, r}(\mathbb{R})\right)$ and $K \in C\left(\mathbb{R}^{+}, M_{d, d}(\mathbb{R})\right)$. In addition, we assume that $K \in L^{1}\left(\mathbb{R}^{+}\right)$.

The existence and uniqueness of a continuous solution of (2.3) is covered in Berger and Mizel [2], for instance. We can also represent the solution of (2.3) in terms of the resolvent of (2.1).

Lemma 2.1. Under the above conditions on $K$ and $\Sigma$, if $X(0)=X_{0}$ is known, there exists a unique a.s. continuous solution to (2.3) on every interval of $\mathbb{R}^{+}$. Moreover, if $Z$ satisfies (2.2), with $Z(0)=I_{d}$, then

$$
X(t)=Z(t) X_{0}+\int_{0}^{t} Z(t-s) \Sigma(s) d W(s)
$$

for all $t \geq 0$.

Proof. The proof follows the line of reasoning in Küchler and Mensch [7], or Mohammed [11].

Remark 2.2 . 
From Lemma 2.1, it is immediate that $X$ is a Gaussian process with expectation vector

$$
m(t)=\mathbb{E} X(t)=Z(t) X_{0}
$$

and covariance matrix

$$
\begin{aligned}
\rho(s, t)=\mathbb{E}\left[(X(s)-\mathbb{E} X(s))(X(t)-\mathbb{E} X(t))^{T}\right] \\
=\int_{0}^{s \wedge t} Z(s-u) \Sigma(u) \Sigma(u)^{T} Z(t-u)^{T} d u .
\end{aligned}
$$

This can be seen by fixing $t$ and adapting the line of argument of Problem 6.2 p.355 in [6]. The main result of this paper establishes the almost sure asymptotic convergence of solutions of (2.3), subject to the zero solution of the underlying deterministic problem (2.1) being asymptotically stable in a specific sense.

We recall the various standard notions of stability of the zero solution required for our analysis; the reader may refer further to Miller [10].

The zero solution of (2.1) is said to be uniformly stable (US), if, for every $\varepsilon>0$, there exists $\delta(\varepsilon)>0$ such that $t_{0} \in \mathbb{R}^{+}$and $\phi \in C\left(\left[0, t_{0}\right] ; \mathbb{R}^{n}\right)$ with $|\phi|_{t_{0}}<\delta(\varepsilon)$ implies $\left\|x\left(t ; t_{0}, \phi\right)\right\|_{1}<\varepsilon$ for all $t \geq t_{0}$. The zero solution is said to be uniformly asymptotically stable (UAS) if is US and there exists $\delta>0$ with the following property: for each $\varepsilon>0$ there exists a $T(\varepsilon)>0$ such that $t_{0} \in \mathbb{R}^{+}$and $\phi \in C\left(\left[0, t_{0}\right] ; \mathbb{R}^{n}\right)$ with $|\phi|_{t_{0}}<\delta$ implies $\left\|x\left(t, t_{0}, \phi\right)\right\|_{1}<\varepsilon$ for all $t \geq t_{0}+T(\varepsilon)$.

The properties of the resolvent $Z$ are deeply linked to the stability of the zero solution of $(2.1)$. It is shown in [10] that the zero solution of (2.1) is UAS if and only if $Z \in L^{1}\left(\mathbb{R}^{+}\right)$. Moreover, the uniform asymptotic stability of the zero solution also implies that

$$
\lim _{t \rightarrow \infty} Z(t)=0 \text {. }
$$

The asymptotic stability of linear functional differential equations with unbounded delay differs from that of linear equations with bounded delay in an important respect: uniformly asymptotically stable solutions of equations with unbounded delay need not be exponentially stable.

Indeed, Murakami [13], [14] has shown when the zero solution is uniformly asymptotically stable (under the restriction that the entries of $K$ do not change sign) that a necessary and sufficient condition for the exponential stability of the zero solution of (2.1) is that there be a number $\gamma>0$ such that

$$
\int_{0}^{\infty}\|K(s)\| e^{\gamma s} d s<\infty
$$

A subexponential rate of decay for scalar versions of (2.1) has been established in Appleby and Reynolds [1]. More precisely, it is shown that when $k$ is a positive, continuous and integrable function which satisfies

$$
\lim _{t \rightarrow \infty} \frac{(k * k)(t)}{k(t)}=2 \int_{0}^{\infty} k(s) d s, \quad \lim _{t \rightarrow \infty} \frac{k(t-s)}{k(t)}=1 \text { uniformly in } s>0 .
$$

the solution of

$$
x^{\prime}(t)=-a x(t)+\int_{0}^{t} k(t-s) x(s) d s
$$

obeys

$$
\lim _{t \rightarrow \infty} \frac{x(t)}{k(t)}=\frac{x(0)}{\left(a-\int_{0}^{\infty} k(s) d s\right)^{2}}
$$


Examples of functions continuous, positive and integrable functions $k$ which satisfy (2.8) are those which obey $k(t) \sim C t^{-\alpha}$ as $t \rightarrow \infty$, for some $C>0, \alpha>1$ (or functions of regular variation more generally), or for which $k(t) \sim C e^{-t^{\alpha}}$ as $t \rightarrow \infty$ with $C>0, \alpha \in(0,1)$. A fuller description of the class of subexponential functions which obey (2.8) is contained in [1]. In view of these results, we impose the additional asymptotic constraint on the resolvent $Z$

$$
\|Z(t)\| t^{\alpha} \leq C, \quad \text { for some } C, \alpha>0 .
$$

In particular, if the zero solution of (2.1) is uniformly asymptotically stable, and $K$ obeys (2.7), then (2.10) is true. (2.10) again holds for the scalar problem (2.9), if $k$ satisfying (2.8) has the asymptotic behaviour of any of the functions mentioned in the previous paragraph.

\section{Main Result}

In this section we prove the principal result of the paper, whose proof requires a simple preliminary lemma.

Lemma 3.1. Suppose $z \in C\left(\mathbb{R}^{+} ; \mathbb{R}\right) \cap L^{1}\left(\mathbb{R}^{+}\right)$satisfies $|z(t)| t^{\alpha} \leq C$ for some $C, \alpha>0$ and $f \in C\left(\mathbb{R}^{+} ; \mathbb{R}^{+}\right)$satisfies

$$
\lim _{t \rightarrow \infty} f(t) \log t=0
$$

then

$$
\lim _{t \rightarrow \infty} \log t \int_{0}^{t} z(t-s)^{2} f(s) d s=0 .
$$

Proof. Since $z(t) \in L^{1}\left(\mathbb{R}^{+}\right)$, and $z(t) \rightarrow 0$ as $t \rightarrow \infty$, we have $z(t)^{2} \in L^{1}\left(\mathbb{R}^{+}\right)$. For every $\varepsilon>0$ there is a $T=T(\varepsilon)>0$ such that $|\log (t+2) f(t)|<\varepsilon$, for all $t>T$. Hence, for $t>T$

$$
\begin{array}{rl}
\log (t+2) \int_{0}^{t} z(t-s)^{2} f(s) d s \leq \log (t+2) \int_{0}^{T} z(t-s)^{2} & f(s) d s \\
& +\varepsilon \int_{T}^{t} z(t-s)^{2} \frac{\log (t+2)}{\log (s+2)} d s .
\end{array}
$$

The first term on the righthand side of this estimate has zero limit as $t \rightarrow \infty$, since $\lim _{t \rightarrow \infty} z(t)^{2} \log t=$ 0 . Let $\mu \in(0,1)$ so that $\mu<2 \alpha$. For $t>T^{1 / \mu}$, we split the second term on the righthand side of (3.1) and bound it according to

$$
\begin{aligned}
& \int_{T}^{t} z(t-s)^{2} \frac{\log (t+2)}{\log (s+2)} d s \\
& \quad=\int_{T}^{t^{\mu}} z(t-s)^{2} \frac{\log (t+2)}{\log (s+2)} d s+\int_{t^{\mu}}^{t} z(t-s)^{2} \frac{\log (t+2)}{\log (s+2)} d s \\
& \leq C^{2} \int_{T}^{t^{\mu}} \frac{1}{(t-s)^{2 \alpha}} \frac{\log (t+2)}{\log (s+2)} d s+\int_{t^{\mu}}^{t} z(t-s)^{2} \frac{\log (t+2)}{\log (s+2)} d s \\
& \leq C^{2} \frac{\log (t+2)}{\left(t-t^{\mu}\right)^{2 \alpha}} \int_{T}^{t^{\mu}} \frac{1}{\log (s+2)} d s+\int_{t^{\mu}}^{t} z(t-s)^{2} \frac{\log (t+2)}{\log (s+2)} d s .
\end{aligned}
$$

As $\mu<2 \alpha$, the first term on the righthand side has zero limit as $t \rightarrow \infty$; the integrability of $z^{2}$ ensures the same for the second term. Returning to (3.1) with these observations proves the result. 
Theorem 3.2. Let $\Sigma \in C\left(\mathbb{R}^{+} ; M_{d, r}(\mathbb{R})\right)$ and $K \in C\left(\mathbb{R}^{+} ; M_{d, d}(\mathbb{R})\right)$ be integrable. If the zero solution of (2.1) is uniformly asymptotically stable and the resolvent obeys (2.10), then

$$
\lim _{t \rightarrow \infty}\|\Sigma(t)\|^{2} \log t=0
$$

implies

$$
\lim _{t \rightarrow \infty} X(t)=0 \quad \mathbb{P} \text {-a.s. }
$$

Proof. Since the zero solution of (2.1) is uniformly asymptotically stable, we have that $Z$, the resolvent of (2.1), satisfies

$$
\lim _{t \rightarrow \infty} Z(t)=0, \quad Z(t) \in L^{1}\left(\mathbb{R}^{+}\right) .
$$

Hence for all $i=1, \ldots, d, j=1, \ldots, d$ we have

$$
\lim _{t \rightarrow \infty} Z_{i j}(t)=0, \quad Z_{i j}(t) \in L^{1}\left(\mathbb{R}^{+}\right) .
$$

Since $Z(t) \rightarrow 0$ as $t \rightarrow \infty,(2.4)$ of Lemma 2.1 implies that $X(t) \rightarrow 0$ a.s. if and only if

$$
\lim _{t \rightarrow \infty} Y(t)=0
$$

where $Y(t)=\int_{0}^{t} Z(t-s) \Sigma(s) d W(s)$ is a zero mean Gaussian process with covariance matrix given by (2.6). By writing $Y(t)=\sum_{j=1}^{d} Y^{(j)}(t) \mathbf{e}_{j}$ and defining $\Sigma_{j}(t)=\operatorname{Var}\left[Y^{(j)}(t)\right]=\rho_{j j}(t, t)$, we see that $Y^{(j)}(t) \sim \mathcal{N}\left(0, \Sigma_{j}(t)\right)$. From (2.6), we easily obtain

$$
\begin{aligned}
\Sigma_{j}(t) & =\sum_{k=1}^{r} \int_{0}^{t}\left(\sum_{i=1}^{d} Z_{j i}(t-u) \Sigma_{i k}(u)\right)^{2} d u \\
& \leq d \sum_{k=1}^{r} \sum_{i=1}^{d} \int_{0}^{t} Z_{j i}(t-u)^{2} \Sigma_{i k}(u)^{2} d u
\end{aligned}
$$

In addition, from (3.2) and norm equivalence, we also have

$$
\lim _{t \rightarrow \infty} \Sigma_{i k}(t)^{2} \log t=0
$$

for all $i=1, \ldots, d$ and $k=1, \ldots, r$. Next, for $i=1, \ldots, d, j=1, \ldots, d, k=1, \ldots, r$, we define

$$
\Sigma_{i j k}(t)=\int_{0}^{t} Z_{j i}(t-u)^{2} \Sigma_{i k}(u)^{2} d u
$$

Obviously, from (3.5), we have

$$
\lim _{t \rightarrow \infty} \Sigma_{j}(t) \log t=0
$$

provided

$$
\lim _{t \rightarrow \infty} \Sigma_{i j k}(t) \log t=0
$$

is true for all $i, j, k$. This last statement is true from Lemma 3.1 by (3.3), (3.6) and (2.10). Let $h(n)=\sqrt{n}$. We now use (3.7) to show for $n \in \mathbb{N}$ that

$$
\lim _{n \rightarrow \infty} Y(h(n))=0 \quad \text { a.s. }
$$


This in turn follows if we can show, for each fixed $j=1, \ldots, d$, that $Y^{(j)}(h(n)) \rightarrow 0$ as $n \rightarrow \infty$, a.s. Let $\varepsilon>0$. By using the symmetry of the density of a zero mean Gaussian, we have

$$
\begin{aligned}
\mathbb{P}\left[\left|Y^{(j)}(t)\right|>\varepsilon\right] & =2 \mathbb{P}\left[Y^{(j)}(t)>\varepsilon\right] \\
& =2 \frac{1}{\sqrt{2 \pi}} \int_{\varepsilon \sqrt{\Sigma_{j}(t)}}^{\infty} \exp \left(-\frac{1}{2} x^{2}\right) d x \\
& \leq \frac{2}{\sqrt{2 \pi}} \frac{1}{\varepsilon \sqrt{\Sigma_{j}(t)}} \exp \left(-\frac{1}{2} \cdot \frac{\varepsilon^{2}}{\Sigma_{j}(t)}\right),
\end{aligned}
$$

where the last bound follows from Mill's estimate. Since

$$
\lim _{n \rightarrow \infty} \Sigma_{j}(h(n)) \log n=2 \lim _{n \rightarrow \infty} \Sigma_{j}(h(n)) \log h(n)=0,
$$

there exists $N_{j}(\varepsilon) \in \mathbb{N}$ such that, for $n>N_{j}(\varepsilon)$

$$
\Sigma_{j}(h(n))<\frac{\varepsilon^{2}}{4 \log n} .
$$

Thus for $n>N_{j}(\varepsilon)$

$$
\mathbb{P}\left[\left|Y^{(j)}(h(n))\right|>\varepsilon\right] \leq \frac{2}{\sqrt{2 \pi}} \cdot \frac{1}{2 \sqrt{\log n}} \cdot \frac{1}{n^{2}}
$$

SO

$$
\sum_{n=N_{j}(\varepsilon)}^{\infty} \mathbb{P}\left[\left|Y^{(j)}(h(n))\right|>\varepsilon\right]<\infty .
$$

Since the sum from $n=1$ to $n=N_{j}(\varepsilon)-1$ is finite, the first Borel-Cantelli lemma allows us to conclude that $Y^{(j)}(h(n)) \rightarrow 0$ as $n \rightarrow \infty$, a.s., from which (3.8) follows.

At this point we will show that

$$
\lim _{n \rightarrow \infty} \sup _{h(n) \leq t \leq h(n+1)}\|Y(t)\|=0, \quad \text { a.s., }
$$

which, in conjunction with (3.8), proves the theorem. To prove (3.9), we make the following semimartingale decomposition of $Y$ :

$$
Y(t)=\int_{0}^{t} \Sigma(s) d W(s)+\int_{0}^{t}\left(\int_{0}^{s} Z^{\prime}(s-u) \Sigma(u) d W(u)\right) d s .
$$

(The existence of $Z^{\prime}$ is guaranteed by equation (2.2) in Lemma 2.1.) From the above semimartingale decomposition, if $h(n) \leq t \leq h(n+1)$, we have

$$
Y(t)=Y(h(n))+\int_{h(n)}^{t} \Sigma(s) d W(s)+\int_{h(n)}^{t}\left(\int_{0}^{s} Z^{\prime}(s-u) \Sigma(u) d W(u)\right) d s .
$$

Showing that

$$
\lim _{n \rightarrow \infty} \max _{h(n) \leq t \leq h(n+1)}\left\|\int_{h(n)}^{t} \Sigma(s) d W(s)\right\|=0, \quad \text { a.s. }
$$

and

$$
\lim _{n \rightarrow \infty} \max _{h(n) \leq t \leq h(n+1)}\left\|\int_{h(n)}^{t}\left(\int_{0}^{s} Z^{\prime}(s-u) \Sigma(u) d W(u)\right) d s\right\|=0, \quad \text { a.s., }
$$

suffices to prove (3.9). 
We first deal with the second term on the right hand side of (3.10); its expected value can be bounded by the vector version of the Burkholder-Davis-Gundy inequality (see for example, [6]), to wit:

$$
\begin{aligned}
\mathbb{E}\left[\max _{h(n) \leq t \leq h(n+1)}\left\|\int_{h(n)}^{t} \Sigma(s) d W(s)\right\|^{8}\right] & \leq \Lambda_{4}\left(\int_{h(n)}^{h(n+1)}\|\Sigma(s)\|_{F}^{2} d s\right)^{4} \\
& \leq \Lambda_{4}\left(\bar{\sigma}^{2} r d\right)^{4}(h(n+1)-h(n))^{4} \\
& \leq \Lambda_{4}\left(\bar{\sigma}^{2} r d\right)^{4} \frac{1}{2^{4}} \frac{1}{n^{2}}
\end{aligned}
$$

where $\Lambda_{4}>0$ is independent of $t$ and $n$, and $\sup _{t \geq 0}\left|\Sigma_{i j}(t)\right| \leq \bar{\sigma}<\infty$, for all $i, j=1, \ldots, d$. (The existence of such an $\bar{\sigma}$ follows from (3.2) and the continuity of $\Sigma$ ). By Chebyshev's inequality, the sequence

$$
\mathbb{P}\left[\max _{h(n) \leq t \leq h(n+1)}\left\|\int_{h(n)}^{t} \Sigma(s) d W(s)\right\|>\varepsilon\right]
$$

is summable for every $\varepsilon>0$, so by the first Borel-Cantelli Lemma, we conclude that (3.11) holds.

To prove (3.12), we use the Cauchy-Schwartz inequality and Jensen's inequality to obtain

$$
\begin{aligned}
& \mathbb{E}\left[\sup _{h(n) \leq t \leq h(n+1)}\left\|\int_{h(n)}^{t}\left(\int_{0}^{s} Z^{\prime}(s-u) \Sigma(u) d W(u)\right) d s\right\|^{8}\right] \\
& \leq \mathbb{E}\left[\sup _{h(n) \leq t \leq h(n+1)}(t-h(n))^{7} \int_{h(n)}^{t}\left\|\int_{0}^{s} Z^{\prime}(s-u) \Sigma(u) d W(u)\right\|^{8} d s\right] \\
& =(h(n+1)-h(n))^{7} \int_{h(n)}^{h(n+1)} \mathbb{E}\left\|\int_{0}^{s} Z^{\prime}(s-u) \Sigma(u) d W(u)\right\|^{8} d s .
\end{aligned}
$$

The last equality is justified by Fubini's theorem.

For any $s \geq 0$, define

$$
I(s)=\int_{0}^{s} Z^{\prime}(s-u) \Sigma(u) d W(u)
$$

so that

$$
\begin{array}{r}
\mathbb{E}\left[\sup _{h(n) \leq t \leq h(n+1)}\left\|\int_{h(n)}^{t}\left(\int_{0}^{s} Z^{\prime}(s-u) \Sigma(u) d W(u)\right) d s\right\|^{8}\right] \\
\leq(h(n+1)-h(n))^{7} \int_{h(n)}^{h(n+1)} \mathbb{E}\|I(s)\|^{8} d s .
\end{array}
$$

Therefore, we merely require a bound on $\mathbb{E}\|I(s)\|^{8}$. In view of Remark 2.2 , it is clear that $I_{j}(s)=I(s)^{T} \mathbf{e}_{j} \sim \mathcal{N}\left(0, \delta_{j}(s)\right)$. Using the same computation as (3.5), we obtain

$$
\delta_{j}(s) \leq d \sum_{k=1}^{r} \sum_{i=1}^{d} \int_{0}^{s} Z_{j i}^{\prime}(s-u)^{2} \Sigma_{i k}(u)^{2} d u .
$$


Since by Lemma 3.3, the entries of $Z^{\prime}$ are bounded, and the entries of $\Sigma$ are bounded (by $(3.2))$, it follows that $\delta_{j}(s) \leq L_{1} s$ for some $L_{1}>0$. Now

$$
\begin{aligned}
\mathbb{E}\|I(s)\|^{8} & =\mathbb{E}\left[\left(\sum_{j=1}^{d} I_{j}(s)^{2}\right)^{4}\right] \leq \mathbb{E}\left[d^{3} \sum_{j=1}^{d} I_{j}(s)^{8}\right] \\
& =d^{3} \sum_{j=1}^{d} \frac{8 !}{2^{4} 4 !} \delta_{j}(s)^{4} \leq d^{3} \frac{8 !}{2^{4} 4 !}\left(L_{1} s\right)^{4}=L_{2} s^{4} .
\end{aligned}
$$

Thus, returning to (3.13), we obtain

$$
\begin{aligned}
& \mathbb{E}\left[\sup _{h(n) \leq t \leq h(n+1)}\left\|\int_{h(n)}^{t}\left(\int_{0}^{s} Z^{\prime}(s-u) \Sigma(u) d W(u)\right) d s\right\|^{8}\right] \\
& \leq(h(n+1)-h(n))^{7} \int_{h(n)}^{h(n+1)} L_{2} s^{4} d s \\
& \leq L_{2}(h(n+1)-h(n))^{8} h(n+1)^{4} \leq \frac{L_{3}}{n^{2}}
\end{aligned}
$$

for some $L_{3}>0$. From Chebyshev's inequality and the above, the sequence of probabilities

$$
\mathbb{P}\left[\sup _{h(n) \leq t \leq h(n+1)}\left\|\int_{h(n)}^{t}\left(\int_{0}^{s} Z^{\prime}(s-u) \Sigma(u) d W(u)\right) d s\right\|>\varepsilon\right]
$$

is summable for any $\varepsilon>0$, so by the first Borel-Cantelli Lemma, we have (3.12).

Using the results (3.8), (3.11), (3.12), it is now immediate from (3.10) that (3.4) is true, and hence the theorem.

It just remains to sketch the proof of the deferred result that the entries of $Z^{\prime}$ are bounded.

Lemma 3.3. If the zero solution of (2.1) is uniformly asymptotically stable, there exists $\Gamma>0$ such that

$$
\left\|Z_{i j}^{\prime}(t)\right\| \leq \Gamma
$$

for all $t \geq 0, i=1, \ldots, d, j=1, \ldots, d$.

Proof. By the uniform asymptotic stability of the zero solution of (2.1), we have (3.3), so by (2.2), we have $Z^{\prime}(t) \rightarrow 0$ as $t \rightarrow \infty$. Moreover, $t \mapsto Z^{\prime}(t)$ is continuous, by the continuity of $K$ and $Z$. Therefore each entry of $Z^{\prime}(t)$ is a bounded function on $\mathbb{R}^{+}$.

Remark 3.4. A careful reading of the proof of Theorem 3.2 reveals that when (3.2) holds that it is sufficient that the resolvent $Z$ satisfies

$$
\lim _{t \rightarrow \infty} Z(t)=0, \quad Z(t) \in L^{2}\left(\mathbb{R}^{+}\right),
$$

for conclusion of Theorem 3.2 to hold. It is possible to construct Volterra equations of the form (2.1) for which (3.14) holds, which do not have uniformly asymptotically stable solutions. 


\section{NeCESsity of the CONDition (3.2)}

In this section, we examine in more detail the role of the condition (3.2) in the almost sure asymptotic stability of the solution of (2.3). In particular, we show for the general finitedimensional version of (2.3) under ceratin monotonicity conditions on $\Sigma$ that the a.s. asymptotic stability of the solution of (2.3) implies (3.2). Under some mild conditions, therefore, the rate of decay of the noise implied by (3.2) is not merely sufficient for the almost sure asymptotic stability of solutions of (2.3), but also necessary.

To prove this result, we need the following fact, which is a simple corollary of a more general theorem of Chan [3, Theorem 1].

Lemma 4.1. Suppose that $\Sigma \in C\left(\mathbb{R}^{+} ; \mathbb{R}^{d} \times \mathbb{R}^{r}\right)$ such that $t \mapsto\|\Sigma(t)\|^{2}$ is a decreasing function with $\lim _{t \rightarrow \infty}\|\Sigma(t)\|=0$. Let $a>0$. If $(W(t))_{t \geq 0}$ is a $r$-dimensional Brownian motion, and the solution of

$$
d Y(t)=-a Y(t) d t+\Sigma(t) d W(t)
$$

obeys $\lim _{t \rightarrow \infty} Y(t)=0$, a.s., then

$$
\int_{0}^{\infty} \exp \left(-c\|\Sigma(s)\|^{-2}\right) d s<\infty \quad \text { for all } c>0 .
$$

We observe when $\|\Sigma\|$ is decreasing that (4.2) is equivalent to (3.2), as pointed out in Chan and Williams [4, Theorem 1]. Given this observation, we can prove a converse result to Theorem 3.2 .

Theorem 4.2. Let $\Sigma \in C\left(\mathbb{R}^{+} ; M_{d, r}(\mathbb{R})\right)$, and suppose $t \mapsto\|\Sigma(t)\|^{2}$ is decreasing with $\lim _{t \rightarrow \infty}\|\Sigma(t)\|=$ 0 . If $K \in C\left(\mathbb{R}^{+} ; M_{d, d}(\mathbb{R})\right)$ is integrable, and the solution of (2.3) satisfies

$$
\lim _{t \rightarrow \infty} X(t)=0 \quad \mathbb{P} \text {-a.s. }
$$

then $\Sigma$ satisfies (3.2).

Proof. Let $a>0$ and define $F(t)=\left(a I_{d}+A\right) X(t)+\int_{0}^{t} K(t-s) X(s) d s$. Since $X(t) \rightarrow 0$ as $t \rightarrow 0$ a.s., and $K$ is integrable, it follows that $F(t) \rightarrow 0$ as $t \rightarrow 0$ a.s. Next, (2.3) can be rewritten as

$$
d X(t)=(-a X(t)+F(t)) d t+\Sigma(t) d W(t)
$$

Define the $\mathbb{R}^{d}$-valued process $(Y(t))_{t \geq 0}$ by $Y(t)=e^{-a t} \int_{0}^{t} e^{a s} \Sigma(s) d W(s)$. Using integration by parts on (4.3) and then rearranging yields

$$
Y(t)=X(t)-X(0) e^{-a t}-\int_{0}^{t} e^{-a(t-s)} F(s) d s .
$$

Since $X(t), F(t) \rightarrow 0$ as $t \rightarrow 0$, it is true that $Y(t) \rightarrow 0$ as $t \rightarrow 0$ a.s. But $Y$ satisfies the stochastic differential equation (4.1), and so the result of Lemma 4.1 applies. According to the remark following Lemma $4.1,\|\Sigma\|$ satisfies (3.2).

\section{Extensions}

We briefly comment on extending the results of this note to linear stochastic functional differential equations with bounded delay, and to linear stochastic neutral difference differential equations. It is evident that the results of this paper can be extended to nonconvolution linear Itô-Volterra equations when the underlying deterministic problem is exponentially stable. 
5.1. Equation with bounded delay. Consider the linear functional differential equation

$$
x^{\prime}(t)=A x(t)+\int_{t-\tau}^{t} K(t-s) x(s) d s, \quad t \geq 0
$$

and its stochastic perturbation

$$
d X(t)=\left(A X(t)+\int_{t-\tau}^{t} K(t-s) X(s) d s\right) d t+\Sigma(t) d W(t)
$$

with initial condition $X(t)=\psi(t)$ for $t \in[-\tau, 0]$, where $\psi \in C\left([-\tau, 0] ; \mathbb{R}^{d}\right)$, and $A, \Sigma$ have the same properties as before, while $K$ need only be defined on $[-\tau, 0]$. A variation of parameters solution of (5.2) similar to (2.4) may be developed in terms of the fundamental solution of (5.1), which is defined by

$$
\begin{gathered}
Z^{\prime}(t)=A Z(t)+\int_{t-\tau}^{t} K(t-s) Z(s) d s, \quad t \geq 0 \\
Z(t)=0, t \in[-\tau, 0), \quad Z(0)=I_{d} .
\end{gathered}
$$

In this case however, the uniform asymptotic stability of the zero solution of (5.1) is equivalent to the exponential stability of the zero solution of (5.2). This in turn implies that there is $\alpha>0$ and $C>0$ such that $\|Z(t)\| \leq C e^{-\alpha t}, t \geq 0$. Therefore, condition (2.10) is satisfied, and we have the following result.

Theorem 5.1. Let $\Sigma \in C\left(\mathbb{R}^{+} ; M_{d, r}(\mathbb{R})\right), K \in C\left([-\tau, 0] ; M_{d, d}(\mathbb{R})\right)$, and suppose $t \mapsto\|\Sigma(t)\|^{2}$ is decreasing with $\lim _{t \rightarrow \infty}\|\Sigma(t)\|=0$.

If the zero solution of (5.1) is uniformly asymptotically stable, then

(i) $\|\Sigma\|$ obeys (3.2)

(ii) All solutions of (5.2) satisfy $\lim _{t \rightarrow \infty} X(t)=0$ a.s.

are equivalent.

It is evident that a similar result can be developed for equations which has both distributed and fixed delays, such as

$$
d X(t)=\left(A X(t)+\sum_{j=1}^{n} A_{j} X\left(t-\tau_{j}\right)+\int_{0}^{\tau} K(s) X(t-s) d s\right) d t+\Sigma(t) d W(t) .
$$

5.2. Neutral equations. It is possible also to study linear neutral stochastic differential difference equations, for instance

$$
d(X(t)-C X(t-\tau))=(A X(t)+B X(t-\tau)) d t+\Sigma(t) d W(t),
$$

where $A, B, C \in M_{d, d}(\mathbb{R})$. Once again, we require that the zero solution of the deterministic version of this equation, viz.,

$$
\frac{d}{d t}(x(t)-C x(t-\tau))=A x(t)+B x(t-\tau) .
$$

is uniformly asymptotically stable. A result such as Theorem 5.1 holds in this case also.

Acknowledgement It is a pleasure to thank David Reynolds for motivating this problem. I am also grateful to the paper's referee for pointing out some simplifications to the proofs. 


\section{REFERENCES}

[1] J. A. D. Appleby and D. W. Reynolds. Subexponential solutions of linear Volterra integro-differential equations and transient renewal equations. Proc. Roy. Soc. Edinburgh. Sect. A, 132:521-543, 2002.

[2] M. A. Berger and V. J. Mizel. Volterra equations with Itô integrals I. Journal of Integral Equations, $2(3): 187-245,1980$.

[3] T. Chan. On multi-dimensional annealing problems. Math. Proc. Cambridge Philos. Soc., 105(1):177-184, 1989.

[4] T. Chan and D. Williams. An "excursion" approach to an annealing problem. Math. Proc. Cambridge Philos. Soc., 105(1):169-176, 1989.

[5] R. D. Driver. Existence and stability of solutions of a delay differential system. Arch. Rational Mech. Anal., 10:401-426, 1962.

[6] I. Karatzas and S. E. Shreve. Brownian Motion and Stochastic Calculus, volume 113 of Graduate Texts in Mathematics. Springer, New York, 1991.

[7] U. Küchler and S. Mensch. Langevin's stochastic differential equation extended by a time-delay term. Stochastics Stochastics Rep., 40(1-2):23-42, 1992.

[8] X. Mao. Almost sure exponential stability of delay equations with damped stochastic perturbation. Stochastic Analysis and Applications, 19(1):67-84, 2001.

[9] X. Mao and X. Liao. Almost sure exponential stability of neutral differential difference equations with damped stochastic perturbations. Electronic Journal of Probability, 1:1-16, 1996.

[10] R. K. Miller. Asymptotic stability properties of linear Volterra integrodifferential equations. Journal of Differential Equations, 10:485-506, 1971.

[11] S.-E. A. Mohammed. Stochastic Functional Differential Equations, volume 99 of Research Notes in Mathematics. Pitman, London, 1984.

[12] S.-E. A. Mohammed and M. K. R. Scheutzow. Lyapunov exponents and stationary solutions for affine stochastic delay differential delay equations. Stochastics and Stochastics Reports, 29:259-283, 1990.

[13] S. Murakami. Exponential stability for fundamental solutions of some linear functional differential equations. In T. Yoshizawa and J.Kato, editors, Proceedings of the international symposium: Functional differential equations, pages 259-263, Singapore, 1990. World Scientific.

[14] S. Murakami. Exponential asymptotic stability of scalar linear Volterra equations. Differential Integral Equations, 4(3):519-525, 1991. 